\title{
Cosmic-ray detection with and novel reconstruction algorithms for the ARIANNA experiment
}

\author{
Anna Nelles* for the ARIANNA Collaboration ${ }^{\dagger}$ \\ ECAP, Friedrich-Alexander-Universität Erlangen-Nürnberg, 91058 Erlangen, Germany \\ DESY, Platanenallee 6, 15738, Zeuthen, Germany \\ E-mail: anna.nelles@desy.de
}

The ARIANNA experiment in Antarctica is targeted at detecting the radio emission of neutrino interactions in the ice. However, due to their antennas being deployed close to the surface, ARIANNA stations have routinely been used for cosmic ray detection. These fully self-triggered events have in-turn been used to improve reconstruction algorithms and to provide a proof-ofprinciple for neutrino detection and reconstruction. We will present the detected events and their characteristics. Also, we will elaborate on novel algorithms to reconstruct the cosmic-ray energy from a single station and to reconstruct the signal polarization from distributed antennas. While necessary for sparse neutrino arrays, these methods also provide new opportunities for stand-alone cosmic ray arrays to improve event statistics and reconstruction quality.

36th International Cosmic Ray Conference -ICRC2019-

July 24th - August 1st, 2019

Madison, WI, U.S.A.

\footnotetext{
${ }^{*}$ Speaker.

${ }^{\dagger}$ for collaboration list see PoS(ICRC2019)1177
} 


\section{Introduction}

The ARIANNA experiment is located on the Ross-Ice-Shelf in Antarctica. In a uniquely radio quiet area, a hexagonal array of pilot-stations has been taking data for several years. Some of the stations have been retrofitted to address different $R \& D$ needs instead of just showing their performance with respect to neutrino detection [3], such as one station being powered by a wind turbine [5]. These proceedings uses a station that has been adapted to measure cosmic rays. Results from another set-up sensitive to air shower signals are discussed in [6].

In distributed neutrino arrays, the spacing between antenna stations will always be large to avoid an overlap in effective volume for neutrino detection. These distances are typically larger than the radio footprint from an air shower, unless very horizontal showers are considered. However, it is useful to foresee upward facing antennas at each station, to detected air showers both for a veto to avoid signal confusion and for calibration purposes. For both these goals, ideally the shower is reconstructed. However, as the spacing between station is so large, air-shower characteristics have to be reconstructed from a single station, which requires new algorithms as opposed to what airshower radio arrays are using. Also, the typical neutrino detector covers a much wider frequency range, which provides new opportunities. These proceedings summarize our methods and findings with ARIANNA and elaborate on the consequences for future neutrino and cosmic-ray arrays.

\section{Cosmic rays identification with ARIANNA}

As shown in [1], the ARIANNA hardware makes it easy to distinguish cosmic rays from background, due to the behaviour of the hardware and the characteristic broad-band frequency spectrum of cosmic rays. Cut criterion is the signal amplitude and the correlation with simulated signal templates. A correlation of better than 0.4 is needed to be considered as candidate and for large signals one needs correlations of better than 0.7. The analysis presented in [1] was repeated with the same cuts, after the cosmic ray station had been upgraded to have four upward pointing logperiodic dipole antennas (LPDA), optimized for cosmic-ray reconstruction, instead of two upward tilted antennas as before the upgrade.

The set of measured cosmic rays is therefore not significantly larger than previously published (i.e. only twice the previously reported time was covered), which would warrant an update to the spectrum measurement, but different with respect to event quality. We will use this set to test our methods for event reconstruction and compare to simulation. It should also be noted that the present set was not optimized for event purity and a small contamination with non-cosmic ray events is likely. We have marked those events that show clear characteristics (RF line contamination, doublepulse structure) of not being caused by an air shower. An analysis that optimizes purity will be performed in the future analogously to [3], once a sufficiently large data-set has been gathered.

\section{Reconstruction methods}

The methods presented here, were developed based on the following assumptions: the signals are detected in a broad-range of frequencies, at least $80-300 \mathrm{MHz}$. There is only the detection in one single station, consisting of two orthogonal pairs of upward facing LPDAs. All additional 

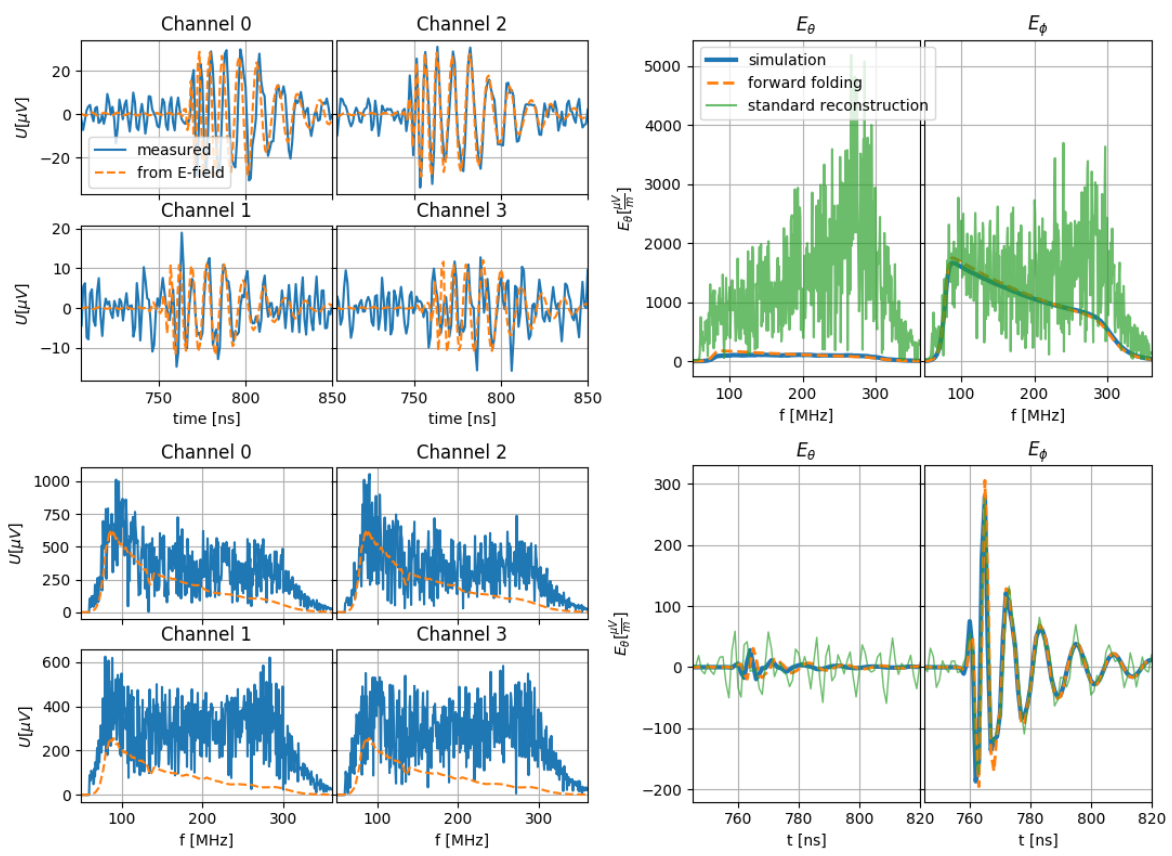

Figure 1: Comparison of the forward folding algorithm with the standard unfolding (from [2]). The top left four panels show the measured voltages in four antennas and those reconstructed through the forward folding. The bottom left four panels show the corresponding frequency spectrum. The right side shows the simulated electric field (without noise), the one obtained through forward folding and the one of obtained from the standard unfolding. The top panels show frequency and the bottom panels the time domain of the two polarization components of the electric field.

information, such as more antennas per station, the detection in multiple stations or the existence of for example a particle detector, allowing for a hybrid-reconstruction, will only improve the reconstruction results presented here. They do not impact the ability to the use the algorithms.

\subsection{Forward-folding to reconstruct the electric field}

Unfolding the effect of instrumentation hardware in the presence of noise has been shown to introduce a bias. For frequencies or arrival directions, where the system is not very sensitive, no signal should be measured. However, due to noise a signal is measured, meaning that if the measured signal were due to a real physical, the signal must have been large. In other words, a small signal is divided by an even smaller sensitivity, vastly overestimating the original signal as unfolding the hardware response is equal to dividing by the hardware response in the frequency domain. This has been found to be especially problematic with respect to two problems: The reconstruction of the signal polarization, in cases where very horizontal showers are also only measured in two horizontal antennas and the reconstruction of the frequency spectrum of the signal at high frequencies, when the system response is less sensitive to high frequencies and at the same time the signal is weak.

To counteract the problem, we have developed the forward-folding algorithm [2]. We assume that every air shower pulse can be modelled analytically by two amplitudes, a frequency slope and a phase between the two polarization directions. We then forward-fold this analytic pulse with the 
hardware response and compare to the measurement not in the physical quantity of the electric field, but in the measured voltage traces. One can then optimize the signal parameters and obtain a noise-free estimate of the original pulse as shown in Fig. 1. As expected this algorithm is much less biased by noise and well suited for the reconstruction of air shower parameters which is discussed in the following sections.

We would like to note that this algorithm delivers the full electric field from distributed antennas, as it also corrects for time offsets due to spacial separation of the antennas. As it can be quite complex to construct an antenna that is sensitive to three polarization directions, but only measuring all three polarization directions ensures a good quality of reconstruction, one should consider employing this algorithm and combine different antennas in software rather than in hardware.

\subsection{Polarization reconstruction}

As the forward-folding algorithms fits the amplitude of the two signal polarizations, it directly fits the polarization of the signal. For the detected air showers the polarization is a suitable indicator to confirm whether an event is truly an air shower due to the dominance of the geomagnetic effect and thereby the main polarization direction.

For neutrino signals, the polarization direction is important because it is needed to reconstruct the neutrino arrival direction. In ice, the signal arrival direction is not co-aligned with the shower axis, as it is the case for air showers. As the Cherenkov angle in ice is typically $\sim 54^{\circ}$, knowing only the arrival direction of the signal, leads to a large uncertainty in the neutrino arrival direction (see also [4]). As the emission is, however, solely due to the Askaryan effect, the polarization vector of the signal, will point towards the shower axis, therefore breaking the degeneracy. Thus, the performance of the reconstruction is both relevant to confirm the detection of cosmic rays and to understand the reconstruction uncertainties for neutrinos.
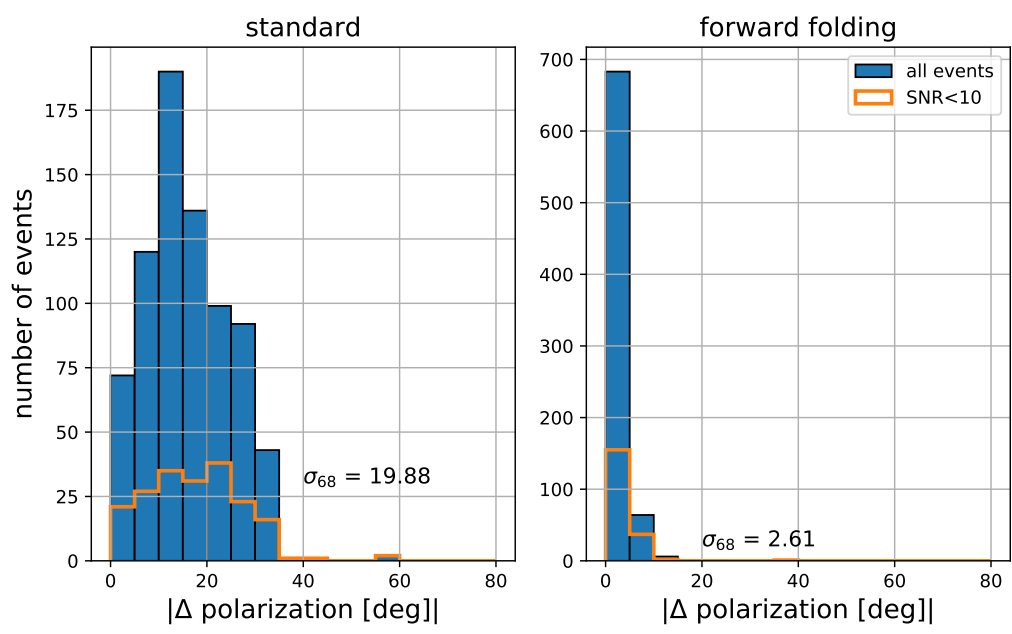

Figure 2: Polarization reconstruction as obtained from CoREAS simulations using the standard unfolding (left) and the forward folding (right). The reconstructed polarization is compared to the $\vec{v} \times \vec{B}$-polarization of the shower. The prediction also includes noise and an estimate for systematic uncertainties.

Figure 2 shows the expected performance of the standard unfolding and the forward folding using four displaced LPDAs to reconstruct the shower polarization. For the reconstruction a slightly 

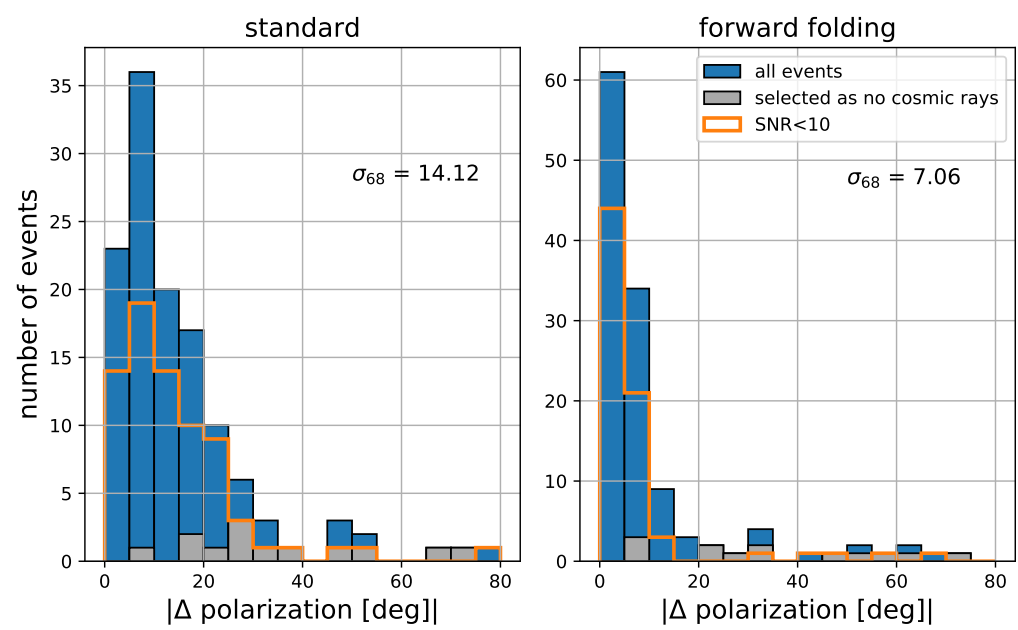

Figure 3: Polarization reconstruction as obtained from cosmic rays measured with ARIANNA, same as Fig. 2. It should be noted that outliers could be a contamination of the set with non-cosmic rays, as the set was not optimized for purity. After the event selection, a number of events were identified by eye based on their waveform characteristics as non-cosmic rays. These are shown in grey and cause most, however not all, of the outliers. These events are excluded for the calculation of the $68 \%$ quantile.

different detector is used then for the simulations, to mimic a non-perfect detector response. The antennas are moved by $10 \mathrm{~cm}$ in random directions and the amplifier is taken to be $10 \%$ different. The same detector response is used for all the events. Albeit this being realistically achievable uncertainties, we may err on the optimistic side for the current prototype ARIANNA station. With a more thoroughly calibrated detector, yielding a shift of the antenna position of $3 \mathrm{~cm}$ and a difference for the amplifier of 5\%,68\% quantiles of 8 and $2 \mathrm{deg}$ are obtainable for the standard and forward folding method respectively. One should note that even with a perfect detector description and no noise, the expectation is non-zero, since we do not compare to the true polarization but the simplified $\vec{v} \times \vec{B}$-expectation.

The reconstructed quantities are shown for the detected cosmic rays in Fig. 3. Using the forward folding instead of the standard method increases the accuracy, however not as much as expected. The contamination with non-cosmic rays could explain the outliers in the data. Similar outliers occur in simulations, if the fit of the arrival direction fails to obtain the correct direction. Further studies are needed to better quantify the agreement.

\subsection{Energy reconstruction from a single station}

Reconstructing the energy of an air shower with a radio detector has been shown to be very accurate, less sensitive to hadronic shower-to-shower fluctuations and competitive in systematic uncertainties to optical detection methods [7]. However, it typically requires the detection of the air shower footprint in several stations and the integration of the signal over the reconstructed footprint. In a sparse detector array, this would only be possible for very few showers. We thus developed an algorithm to reconstruct the energy from a measurement in one station. More details can be found in [8]. 
In general, the amplitude of the pulses scales linearly with energy and more complex as function of distance to the shower axis. In the frequency-domain (as shown in Fig. 4), one can see that the amplitude at the lowest frequency remains similar, but that the total amplitude is determined by the high frequencies. Thus, we developed a method that uses fits to the frequency spectrum of the signal to determine the energy of the shower. We correct for the angle to the magnetic field, the distance to the shower maximum (zenith angle effect), and use a parametrization as function of energy fluence (integrated signal power) and frequency slope to obtain the energy.

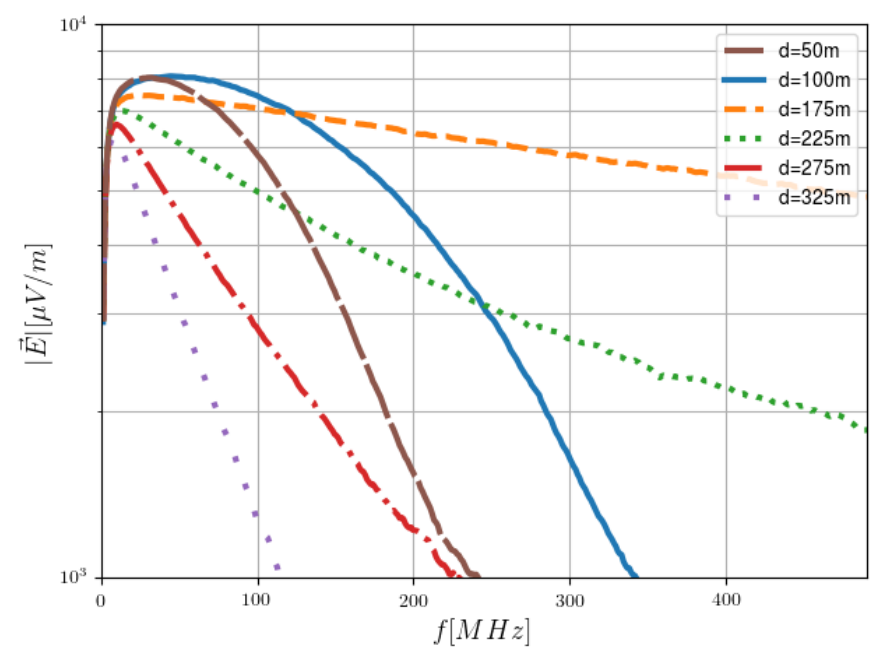

Figure 4: Change in the frequency spectrum of the electric field for an observer at different positions from the shower axis. The signal close to the axis starts steep and somewhat convex until it becomes flat close to the Cherenkov angle. Further out, it falls steeper again until it becomes very steep, showing a hint of concavity. One should note that log-scale of the figure, meaning that a fit of an exponential is still a good approximation in the frequency range above $100 \mathrm{MHz}$.

As opposed to earlier approaches using the signal characteristics [9, 1], this parametrization is valid for all showers and does not rely on dedicated simulations for each event. The energy resolution obtainable is shown in Fig. 5. The resolution is not easily described for vertical showers in polar regions, as here a small footprint coincides with a vertical magnetic field leading to reduced geomagnetic emission. Therefore, vertical showers are excluded from the distribution. The accuracy of the method is best for inclined showers and was tested up to $85^{\circ}$ on simulations.

Fig. 6 shows the energies as reconstructed for the cosmic ray data set. We exclude all those events more inclined than $75^{\circ}$ as above those angles, the refraction/reflections on a snow surface become increasingly difficult to treat [6]. The obtained energies of between $10^{17.3}$ and $10^{18.6} \mathrm{eV}$ correspond to what has been found in [1] and the distribution is mostly dominated by the ARIANNA trigger threshold. A more detailed analysis will need to be performed, including all systematic uncertainties, before an update to the measured spectrum can be presented.

\section{Challenges and opportunities in cosmic-ray detection with neutrino arrays}

The ARIANNA pilot-stage array has shown that one can detect air showers with an array built for neutrino detection. The reconstructed arrival directions, polarizations and energies are 


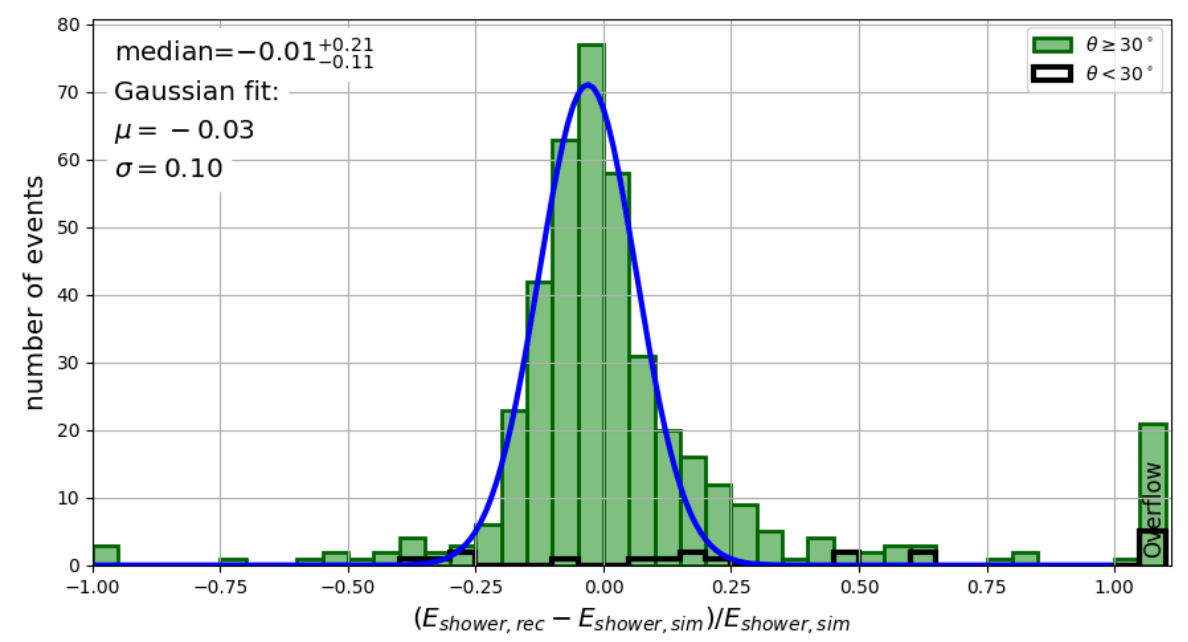

Figure 5: The resolution obtainable with the slope-method as presented in [8]. The overflow bin contains misreconstructed events that can almost exclusively be removed from the set with a higher SNR cut.

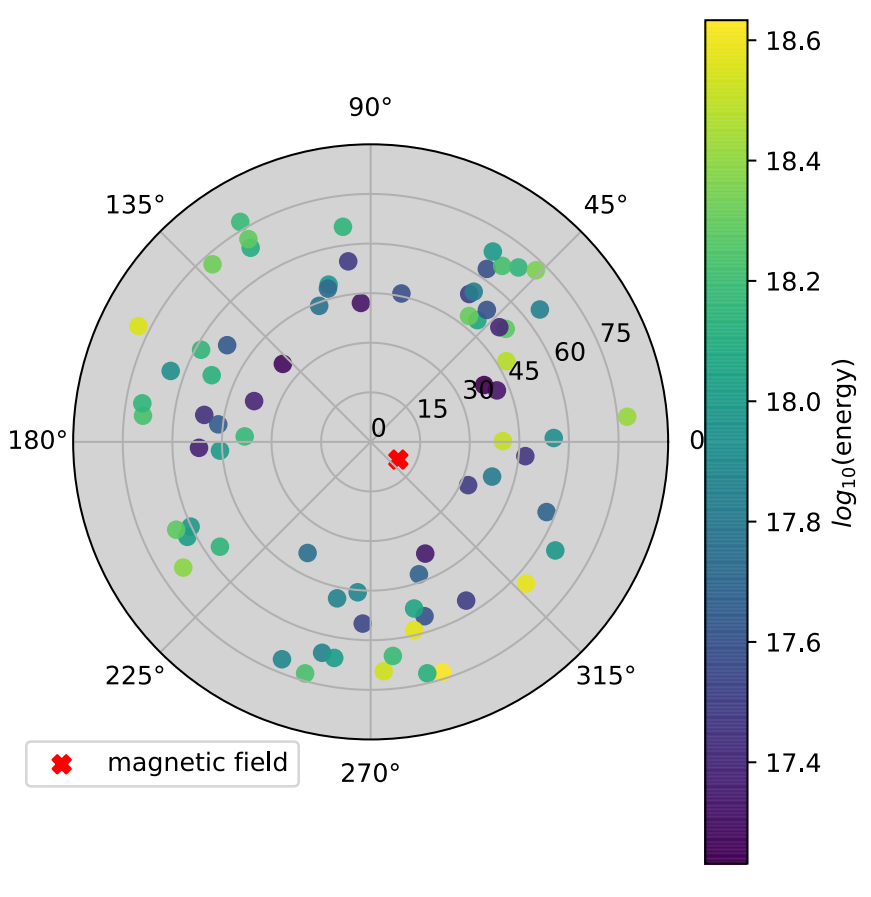

(1).pdf

Figure 6: Reconstructed energies with the slope-method of air showers measured with an ARIANNA station at Moore's Bay. The distribution is determined by the trigger threshold. Vertical showers trigger the stations have a smaller footprint (less likely to hit a station), but stronger signal. Horizontal showers have a wider footprint, but need higher energies to trigger the stations. 
consistent within expectations for such a small array, targeted at showing the readiness for neutrino detection. The large frequency bandwidth of neutrino detectors provides new opportunities for reconstruction that would have been difficult to obtain with the 'classical' bandwidth of 30-80 MHz. The sparseness of neutrino arrays, however, also means that well-established reconstruction techniques based on recorded signals in several stations cannot be used. Instead of fitting an entire air shower footprint sampled in many stations, we derive air shower characteristics from the measurement in a single station. We have discussed new methods that allow us to reconstruct the energy. These methods are also usable at dedicated air shower arrays, providing a new perspective and possibility improved resolution for those arrays.

Neutrino radio arrays will cover large areas. They need to veto cosmic rays as confusion background, which is most reliably done by detecting air showers and making sure that all are accounted for. This requires a thorough reconstruction, thereby gathering a significant air shower data-set allowing to compare to other arrays. Since the radio emission of air showers is well understood, measuring air shower provides a reliable calibration signal for neutrino arrays, allowing for proof-of-principle measurements to develop the neutrino reconstruction capabilities.

\section{Acknowledgements}

We acknowledge funding from the German research foundation (DFG) under grants GL 914/11 and NE 2031/2-1 that supported the cosmic ray work done at ARIANNA. We are grateful to the U.S. National Science Foundation-Office of Polar Programs, the U.S. National Science FoundationPhysics Division (grant NSF-1607719) for granting the ARIANNA array at Moore's Bay.

\section{References}

[1] S. W. Barwick et al., Radio detection of air showers with the ARIANNA experiment on the Ross Ice Shelf, Astroparticle Physics 90 (2017) 50-68

[2] C. Glaser et al., NuRadioReco: a reconstruction framework for radio neutrino detectors, Eur. Phys. J. C (2019) 79: 464

[3] C. Persichilli et al. Performance of the ARIANNA pilot array, and implications for the next generation of UHE neutrino detectors, PoS(ICRC2019)980

[4] C. Glaser for the ARIANNA Collaboration, Neutrino direction and energy resolution of Askaryan detectors, PoS(ICRC2019)899

[5] A. Nelles for the ARIANNA Collaboration, A wind-turbine for autonomous stations for radio detection of neutrinos, PoS(ICRC2019)968

[6] S. H. Wang for the ARIANNA and TAROGE Collaborations, Status, Calibration, and Cosmic Ray Detection of ARIANNA-HCR Station, PoS(ICRC2019)462

[7] The Pierre Auger Collaboration, Energy estimation of cosmic rays with the Engineering Radio Array of the Pierre Auger Observatory, Phys. Rev. D 93 (12) (2016)

[8] C. Welling et al., Reconstructing the cosmic-ray energy from the radio signal measured in one single station, submitted to JCAP, arXiv:1905.11185

[9] H. Schoorlemmer et al., Energy and flux measurements of ultra-high energy cosmic rays observed during the first ANITA flight, Astropart. Phys. 77 (2016) 32-43 